International Journal of Engineering \& Technology, $7(4.10)(2018) 410-412$
International Journal of Engineering \& Technology
SPC
Website: www.sciencepubco.com/index.php/IJET
Research paper

\title{
Analysis of Lung Tumour Detection and Segmentation Using Level Set Method of Active Contour Model
}

\author{
K.Gopi ${ }^{1 *}$, J.Selvakumar ${ }^{2}$ \\ ${ }^{1}$ Dept. of ECE, SRM University, Chennai, India \\ ${ }^{2}$ Dept. of ECE, SRM University, Chennai, India \\ *Corresponding author E-mail: gopi.k@ktr.srmuniv.ac.in
}

\begin{abstract}
Lung cancer is the most common leading cancer in both men and women all over the world. Accurate image segmentation is an essential image analysis tool that is responsible for partitioning an image into several sub-regions. Active contour model have been widely used for effective image segmentation methods as this model produce sub-regions with continuous boundaries. It is used in the applications such as image analysis, deep learning, computer vision and machine learning. Advanced level set method helps to implement active contours for image segmentation with good boundary detection accuracy. This paper proposes a model based on active contour using level set methods for segmentation of such lung CT images and focusing 3D lesion refinement. The features were determined by applying a multi-scale Gaussian filter. This proposed method is able to detect lung tumors in CT images with intensity, homogeneity and noise. The proposed method uses LIDC-IDRI dataset images to segment accurate 3D lesion of lung tumor CT images.
\end{abstract}

Keywords: Image segmentation, LIDC-IDRI dataset, active contours, level set methods, multi-scale Gaussian filter.

\section{Introduction}

Lung tumor is one of the dangerous diseases that lead to death for both men and women in the world. The huge part of occurrences of tumor disease are caused due to smoke and lesser percentage of cases are affected in this disease who are not smoked [1].The tumor can be visible in different locality with dissimilar intensities and different shapes and sizes. As an outcome, accurate image segmentation of lung tumor is a challenging problem to identify the abnormalities and noise [2]. Medical image analysis is an important tool used to analyze CT images for early clinical diagnosis, pulmonary diseases, and treatment planning. The predicted tumor growth of data provides the requirement for automatic computerized segmentation [3].

Computer aided diagnosis (CAD) systems helps radiologist to detect the tumor part in the beginning stage. Various methods have been used in the active contour model for the purpose of tumor segmentation. There are several techniques used for extraction of affected region from the lung CT images. Active contour model is one of the efficient methods for segmentation in lung CT images. This model can be divided as edge-based or region-based segmentation. In the region-based segmentation, the analytical data inside and outside of active contour which influenced its growth and segment the medical image with intensity homogeneity[2]. Level set method of active contour models are extensively used in curve growth application, majorly for medical image segmentation[3].

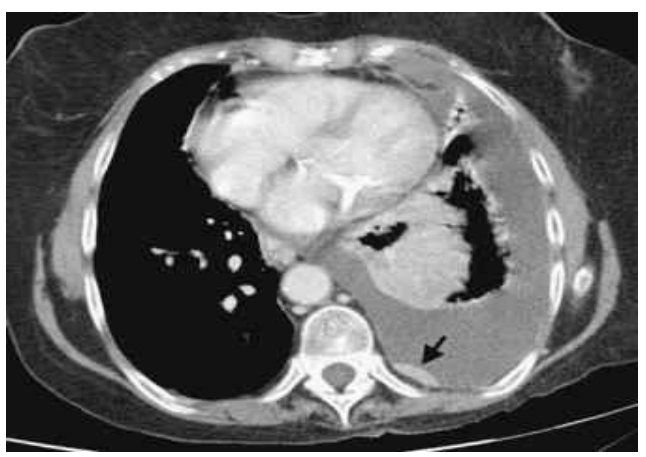

Figure1: Tumor affected lung CT image

\section{Related Work}

Reeves et al. [9] describes about adaptive threshold, which is applied individually for every scan images to compensate for differences between two consecutive lung CT scan. In the lung nodule, midpoint and parenchyma's density was given as the threshold. Hereby, they applied to the geometric constraint to place the segmented nodules in spherical shape while the vessels are removed. Chan et al. [5] proposed a model to detect the affected region using curve evolution techniques. Hereby, they used Mumford-shah functional method for segmentation and continue with level set formulation. This model is used for energy minimization-based segmentation. Herewith they assumed the image that is found two regions of fixed intensity with distinct value. This model detects the objects where the boundaries are not defined by smooth or gradient.

Khadidos et al. [6] explained level set contour method for medical image segmentation. This method is used for the minimization of an energy and those terms are hold with respect to the relative 
importance in the boundary detection. In the weighted level set evolution, we look for different image information such that intensities, textures or edges that helps to give an objective functional. They employed edge information which drives evolving contours to expected boundary. There is ability to converge to the specific boundary in minimum iterations. Farhangi et al. [4] stated that the model preferred the prior shape for sparse linear combinations of training data. In this method, they used Chan-Vese algorithm for region based contour model for curve evolution technique used for their design. This algorithm helps to differentiate the homogeneous part from the region of interest. It requires various steps to draw the curve in the lung tumor. Herewith, they explained the algorithm results such as well circumscribed, pleural tail, juxtavascular and juxta-pleural. They showed the results as relative with manual delineation by different well experienced radiologists Hosseini-Asl et al. [7] describes the segmentation of 3-D lung images based on incremental constrained nonnegative matrix factorization (ICNMF). In this technique, preprocessing has been done to remove the background from an input 3D CT lung image by using region growing method. Using ICNMF algorithm, it explains the structure of $3 \mathrm{D}$ context images. Its performance is comparatively more accurate for each CT input images. This method is performed in both real and synthetic data by the different metrics such as dice similarity coefficient, Absolute Lung Volume Difference (ALVD). This method revealed that the features was robust and encoded the neighbor voxels.

\section{Level Set Active Contour Model}

In the beginning, active contour or snakes methods was used to evolving a curve and subjected to the restriction from a given images, which helps to detect an object in the respective images. The curve has to be drawn around the object which is detected. Then that curve changes its position towards inside and ends on the object boundaries [5].

The Snake model is determined on gradient value of the given image where the edge detector was used. It can be expressed as,

$$
\begin{aligned}
& C_{1}(v)=\alpha \int_{0}^{1}\left|v^{\prime}(s)\right|^{2} d s+\beta \int_{0}^{1}\left|v^{\prime \prime}(s)\right| d s- \\
& \mu \int_{0}^{1}\left|\nabla f_{0}(v(x))\right|^{2} d x
\end{aligned}
$$

where $\alpha, \beta$ and $\mu$ are the positive constant. The First and second term is used to control the contour smoothness (called as internal energy), then the third one which evokes the contour directed the object in the given image (called as external energy) The gradient image $f_{0}$ can be expressed as the equation,

$$
\begin{aligned}
& \lim _{z \rightarrow \infty} g(z)=0 \\
& g\left(\left|\nabla f_{0}(x, y)\right|\right)=\frac{1}{1+\left|\nabla G_{\sigma}(x, y) * f_{0(x, y)}\right|^{p}} p \geq 1
\end{aligned}
$$

Where,

$G_{\sigma} * f_{0}$ is the smooth version of the convolution image $\mathrm{f}_{0}$.

The energy function of the region based active contour model is defined as the

$E^{c v}\left(c, c_{1}, c_{2}\right)=\gamma_{1} \iint_{\operatorname{ins}(c)}\left|I(x, y)-c_{1}\right|^{2} d x d y+\gamma_{2} \iint_{\text {out }(c)}\left|I(x, y)-c_{2}\right|^{2} d x d y+\mu|c|$

Where $\gamma_{1}, \gamma_{2}$ are the constant parameters and in(c),out(c) are the regions inside and outside the contour C. $\mathrm{c}_{1}, \mathrm{c}_{2}$ are the constants that accurate image intensity in the regions ins(c) and out(c).
To determine the features of the tumors with different sizes, Gaussian filter is given with certain range. Then the result is calculated by convolution formula with $\operatorname{SD} \sigma$.

$$
G_{\sigma}(x \cdot y)=G(x \cdot y) * H_{\sigma}(x \cdot y)
$$

Where $H_{\sigma}$ is 2D Gaussian function with SD $\sigma$. The multi-scale Gaussian filter is used for 3D tumor enhancement is explained as,

$$
f_{i}(x, y)=\frac{1}{\sqrt{2 \pi s_{i}^{i}}} \exp \left(-\frac{x^{2}}{2 s_{i}^{2}}\right)-m
$$

From Mumford- shah models, We proposed the energy equation with respect to area and perimeter of the affected tumor region is given as,

$$
\begin{aligned}
E^{A v v}\left(c_{1}, c_{2}, \phi\right) & \\
& =\int_{\Omega}\left(f(x)-c_{1}\right)^{2} H(\phi) d x+\int_{\Omega}\left(f(x)-c_{2}\right)^{2} H_{a}(-\phi) d x+\gamma \int_{\Omega} H_{a}(\phi) d x \\
& +\beta \int_{\Omega} \delta(\emptyset)|\nabla(\emptyset)| d x
\end{aligned}
$$

Where $\phi$ is the curve, $c_{1}$ and $c_{2}$ are the average intensities inside and outside the contour body. The first term and second term refers to the internal and external energy. Then the Third and Fourth term indicates the area and perimeter of the contour part. The unknown minimizing term $\mathrm{u}_{\mathrm{m}}$ as,

$$
\begin{gathered}
u_{m}(x)=c_{1} H(\phi(x))+c_{2}(1-H(\phi(x))) \\
=c_{1} H(\phi(x))+c_{2}(H(-\phi(x)))
\end{gathered}
$$

The energy approximation of the active contour model using level set is expressed as the equation,

$$
\begin{aligned}
B_{a}\left(c_{1}, c_{2}, \phi\right)= & \int_{\Omega}\left(f(x)-c_{1}\right)^{2} H_{a}(\phi) d x+\int_{\Omega}\left(f(x)-c_{2}\right)^{2} H_{a}(-\phi) d x+\gamma \int_{\Omega} H_{a}(\phi) d x \\
& \left.+\beta \int_{\Omega} \delta(\phi) \mid \nabla(\phi)\right) d x
\end{aligned}
$$

Using Euler- Lagrange equation, with respect to the intensities $\mathrm{c}_{1}, \mathrm{c}_{2}$ and $\phi$ are

$$
\begin{aligned}
& c_{1}(\phi)=\frac{\int_{\Omega} f(x) H(\phi(x)) d x}{\int_{\Omega} H(\phi(\mathrm{x})) \mathrm{dx}} \\
& c_{2}(\phi)=\frac{\int_{\Omega} f(x) H(-\phi(x)) d x}{\int_{\Omega} 1-H(\phi(\mathrm{x})) \mathrm{dx}}
\end{aligned}
$$

These are the calculated $c_{1}$ and $c_{2}$ values respectively.

\section{Conclusion}

The proposed algorithm is applied to lung CT images to calculate the result of tumor detection. Our proposed active contour algorithm detects the tumor part in the CT image with intensity homogeneity and reduces the noise precisely. This model extracts the background part from the image. Our method analyzes the results True positive, True negative, false positive and False negative respectively. We use LIDC-IDRI dataset of lung CT images to proceed with our level set active contour models. Then the image 
is segmented with this algorithm having good results in accuracy, specificity and sensitivity.

This model helps to segment the background from the image and introduced the tumor features with energy function and then de- scribed by using multi-scale Gaussian filter. This method having the capable of detect tumor part in the image with reduced noise and intensity homogeneity. Further work can be done with classification algorithms such as SVM for better classification of results.

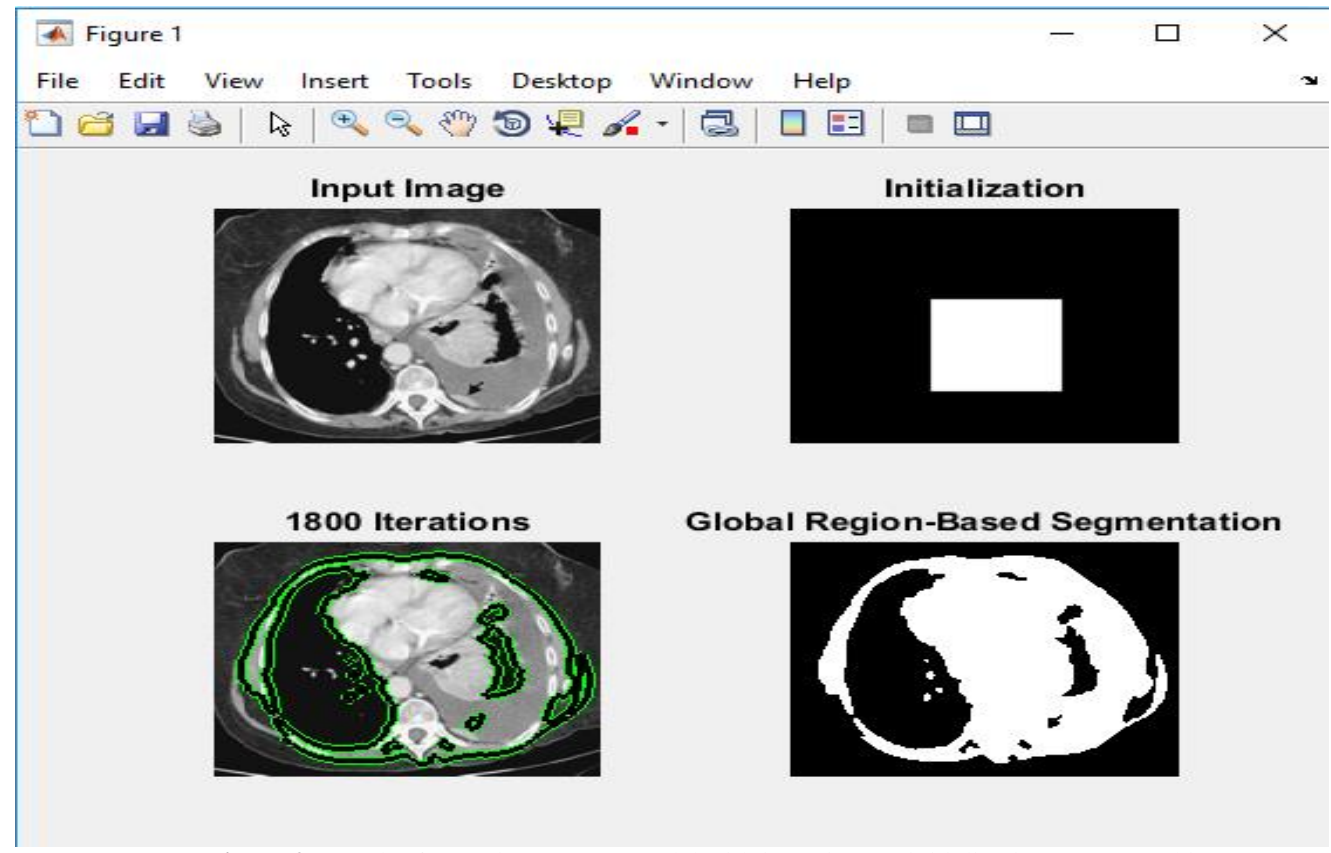

Figure.2: Result of an active contour model with input image, initialization, segmented image.

\section{References}

[1] P.B.Sangamithraa, S.Govindaraju, "Lung tumor detection and classification using EK-mean clustering", IEEE WISPNET 2016 conference.

[2] Maryam Taghizadeh dehkordi, "A new active contour model for tumor segmentation", International conference on pattern recognition and Image analysis april 2017.

[3] Asaaf hoogi, Arjun subramaniam,Rishi veerapaneni, and Daniel L.rubin, "Adaptive estimation of active contour parameters using convolutional neural networks and texture analysis", IEEE transaction on medical imaging march 2017

[4] M.Mehdi Farhangi, Hichem Frigui, Albert seow and amir A Amini, "3-D Active contour segmentation based on sparse linear combination of training shapes", IEEE Transactions on medical imaging, November 2017.

[5] Tony F.Chan and Luminita A.Vese, "Active contours without edges", IEEE transaction on image processing, February 2001.

[6] Alaa Khadidos,Victor sanchez, chang- tsun li, "Weighted level set evolution based on local edge features for medical image segmentation", IEEE transactions on image processing, april 2017.

[7] Ehsan Hosseini- Asl, Jacek M. Zurada, Georgy gimel'farb, and ayman el-baz, " 3-D Lung segmentation by incremental constrained nonnegative matrix factorization",IEEE transactions on biomedical engineering, may 2016.

[8] Mahdi hajiaghayi, Elliott M.Groves, Hamid jafarkhani and Arash kheradvar, "A 3-D Active contour method for automated segmentation of the left ventricle from magnetic resonance images", IEEE transaction on biomedical engineering, January 2017.

[9] A.P.Reeves, A.B. Chan, D.F.Yankelevitz,C.I.Henschke,B.Kressler, and W.J.Kostis, "On measuring the change in size of pulmonary nodules", IEEE transaction on medical imaging, April 2006. 